\title{
Chiral Anomaly and Index Theorem on a finite lattice
}

\author{
Ting-Wai Chiu ${ }^{\text {* }}$
}

'Department of Physics, National Taiwan University, Taipei, Taiwan 106, Republic of China.

The condition for a lattice Dirac operator $D$ to reproduce correct chiral anomaly at each site of a finite lattice for smooth background gauge fields is that $D$ possesses exact zero modes satisfying the Atiyah-Singer index theorem. This is also the necessary condition for $D$ to have correct fermion determinant ( ratio ) which plays the important role of incorporating dynamical fermions in the functional integral.

\section{INTRODUCTION}

Chiral anomaly plays an important role in particle physics. In QCD, the chiral anomaly breaks the global chiral symmetry of massless QCD through the internal quark loop. When the axial current couples to two external photons, the axial anomaly [1] provides a proper account of the decay rate of $\pi^{0} \rightarrow \gamma \gamma$. For the flavor singlet axial current coupling to two gluons, the presence of chiral anomaly but no associated Goldstone boson posed the $U_{A}(1)$ problem which was resolved by 't Hooft, after taking into account of the topologically nontrivial gauge configurations, the instantons [2]. This may also provide an explanation why the $\eta\left(\eta^{\prime}\right)$ particle is much heavier than the $\pi$ 's ( $K$ 's ).

Lattice QCD should be designed to provide nonperturbative and quantitive answers to all these and related problems. However, these goals could not be attained if the lattice Dirac fermion operator does not reproduce correct chiral anomaly or the fermion determinant ( ratio ) on a finite lattice without fine-tuning any parameters.

A basic requirement for lattice Dirac operator $D$ is that, on a finite lattice with any prescribed smooth background gauge field which has integer topological charge, $D$ possesses exact zero modes satisfying the Atiyah-Singer index theorem (i.e., $D$ is topologically proper) without fine tuning any parameters.

"This work was supported by the National Science Council, R.O.C. under the grant numbers NSC88-2112-M002016 and NSC89-2112-M002-017
For any topologically proper $D$, the sum of the anomaly function over all sites must be correct, and is equal to two times of the topological charge of the background gauge field. If the anomaly function of $D$ at some of the sites do not agree with the Chern-Pontryagin density, we can perform the following topologically invariant transformation [3]

$\mathcal{T}(R): \quad D^{\prime}=\mathcal{T}(R)[D] \equiv D(\mathbf{I}+R D)^{-1}$

with some operator $R$ such that $D^{\prime}$ is local, then the anomaly function of $D^{\prime}$ would be in good agreement with the Chern-Pontryagin density at each site ${ }^{2}$. It suffices to choose $R$ to be local in the position space and trivial in the Dirac space. Note that it is not necessary to perform any fine tunings of $R$ since any local $D^{\prime}$ would give the correct chiral anomaly. The set of transformations, $\{\mathcal{T}(R)\}$, form an abelian group with the group parameter space $\{R\}$ [4]. From the physical point of view, we must require the existence of a chirally symmetric $D_{c}=T\left(R_{c}\right)[D]$ such that in the free fermion limit $D_{c}(p) \rightarrow i \gamma_{\mu} p_{\mu}$ as $p \rightarrow 0$, otherwise, $D$ is irrelevant to massless QCD. In general, we assume that there exists

$D_{c}=D\left(\mathbf{I}+R_{c} D\right)^{-1}$

such that $D_{c} \gamma_{5}+\gamma_{5} D_{c}=0$. Then one obtains

$D \gamma_{5}+\gamma_{5} D=-2 D R_{c} \gamma_{5} D$

This is the rejuvenated Ginsparg-Wilson relation [5]. In general, for any $D$, if the chirally

\footnotetext{
${ }^{2}$ Here we have assumed that the size of the finite lattice is large enough such that the finite size effects can be neglected, i.e., the size of the lattice is much larger than the localization length of $D^{\prime}$.
} 
symmetric $D_{c}=\mathcal{T}\left(R_{c}\right)[D]$ exists, then $D$ must satisfy the Ginsparg-Wilson relation. Thus the Ginsparg-Wilson relation does not specify the topological characteristics of a Dirac operator. Only a topologically proper $D$ ( or its transform $\left.D^{\prime}=D(\mathbf{I}+R D)^{-1}\right)$ is suitable for lattice QCD.

\section{THE ANOMALY FUNCTION}

Consider a lattice Dirac operator $D$ which breaks the usual chiral symmetry according to $D \gamma_{5}+\gamma_{5} D=B$, where $B$ is a generic irrelevant operator, and the lattice is finite with periodic boundary conditions. Then we can obtain [4]

$$
\begin{aligned}
\partial^{\mu} J_{\mu}^{5}(x)= & \bar{\psi}_{x} \gamma^{5}(D \psi)_{x}+(\bar{\psi} D)_{x} \gamma^{5} \psi_{x} \\
& -\frac{1}{2}(\bar{\psi} B)_{x} \psi_{x}-\frac{1}{2} \bar{\psi}_{x}(B \psi)_{x}
\end{aligned}
$$

which satisfies the conservation law,

$\sum_{x} \partial^{\mu} J_{\mu}^{5}(x)=0$

If $D$ does not possess exact zero modes in the background gauge field, then the fermionic average of $\partial^{\mu} J_{\mu}^{5}(x)$ is

$$
\left\langle\partial^{\mu} J_{\mu}^{5}(x)\right\rangle=\frac{1}{2} \operatorname{tr}\left[\left(B D^{-1}\right)(x, x)+\left(D^{-1} B\right)(x, x)\right]
$$

$\equiv \mathcal{A}_{n}(x)$ : anomaly function

However, for any $D$ which does not possess exact zero modes in the background gauge field, the sum of the anomaly function over all sites must vanish due to the conservation law,

$\sum_{x}\left\langle\partial^{\mu} J_{\mu}^{5}(x)\right\rangle=\sum_{x} \mathcal{A}_{n}(x)=0$

This implies that if $\mathcal{A}_{n}(x)$ is not zero identically for all $x$, then it must fluctuate from positive to negative values with respect to $x$. The latter case is exactly what happens to the anomaly function of the standard Wilson-Dirac fermion operator.

On the other hand, if $D$ possesses exact zero modes in topologically nontrivial background gauge fields, then $D^{-1}$ is not well defined. In this case, one introduces an infinitesimal mass $m$ and replace $D$ by $\hat{D}=D+m f[D]$ ( where $f[D]$ is any functional of $D$, which has eigenvalue one for the exact zero modes of $D$ ), and finally take the limit $(m \rightarrow 0)$, then we obtain

$$
\begin{aligned}
\left\langle\partial^{\mu} J_{\mu}^{s}(x)\right\rangle= & \mathcal{A}_{n}(x)+2 \sum_{s=1}^{N_{+}}\left[\phi_{s}^{+}(x)\right]^{\dagger} \phi_{s}^{+}(x) \\
& -2 \sum_{t=1}^{N_{-}}\left[\phi_{t}^{-}(x)\right]^{\dagger} \phi_{t}^{-}(x)
\end{aligned}
$$

where $\phi_{s}^{+}$and $\phi_{t}^{-}$are normalized eigenfunctions of $D$ with eigenvalues $\lambda_{s}=\lambda_{t}=0$ and chiralities +1 and -1 respectively ${ }^{3}$, and the anomaly function is

$$
\mathcal{A}_{n}(x)=\lim _{m \rightarrow 0} \frac{1}{2} \operatorname{tr}\left[\left(B \hat{D}^{-1}\right)(x, x)+\left(\hat{D}^{-1} B\right)(x, x)\right]
$$

\section{THE INDEX THEOREM}

Summing Eq. (4) over all sites and using the conservation law Eq. (3), we obtain

$$
N_{-}-N_{+}=\frac{1}{2} \sum_{x} \mathcal{A}_{n}(x)
$$

This is the index theorem for lattice Dirac operator on a finite lattice, in any background gauge field. However, it does not necessarily imply the existence of exact zero modes nor the compliance with the Atiyah-Singer index theorem.

Since $D$ does not have exact zero modes in a trivial gauge background, the index of $D$ must be proportional to $Q$ of the smooth background gauge field. If $Q$ is an integer, then the proportional constant must be an integer, otherwise their product in general cannot be an integer. Denoting this integer multiplier by $c[D]$, we have

$N_{-}-N_{+}=c[D] Q n_{f}$

where $n_{f}$ is number of fermion flavors. Here we have assumed that $c[D]$ is constant for smooth background gauge fields. This is a reasonable assumption since $c[D]$ is an intrinsic characteristics of $D$. However, when the gauge field becomes rough, we expect that the index theorem with integer $c[D]$ would break down. If one insists

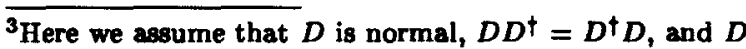
satisfies the hermiticity condition $D^{\dagger}=\gamma_{6} D \gamma_{5}$.
} 
that it holds even for rough gauge configurations, then $c[D]$ cannot be an integer constant due to the highly nonlinear effects of the gauge field. In general, $c[D]$ is a rational number functional of $D$, which in turn depends on the gauge configuration, but it becomes an integer constant only for smooth gauge configurations. The topological characteristics of $D, c[D]$, was first discussed in ref. [6] and was investigated in ref. [7] for the Neuberger operator [8].

\section{A GENERAL SOLUTION FOR $\mathcal{A}_{n}(x)$}

Now we try to obtain a general solution of $\mathcal{A}_{n}(x)$ satisfying (5) and (6), i.e.,

$\frac{1}{2} \sum_{x} \mathcal{A}_{n}(x)=N_{-}-N_{+}=c[D] Q n_{f}$

Consider the gauge configuration with constant field tensors,

$F_{12}^{a}(x)=\frac{2 \pi q_{1}}{L_{1} L_{2}}, \quad F_{34}^{a}(x)=\frac{2 \pi q_{2}}{L_{3} L_{4}}$

and other $F$ 's are zero, where $q_{1}$ and $q_{2}$ are integers. Then the topological charge of this configuration is

$\begin{aligned} Q & =\frac{\operatorname{tr}\left\{t_{a} t_{b}\right\}}{32 \pi^{2}} \sum_{x} \epsilon_{\mu \nu \lambda \sigma} F_{\mu \nu}^{a}(x) F_{\lambda \sigma}^{b}(x+\hat{\mu}+\hat{\nu}) \\ & =n q_{1} q_{2}\end{aligned}$

which is an integer. If $D$ is local, then $\mathcal{A}_{n}(x)$ is constant for all $x$. We immediately obtain

$$
\begin{aligned}
& \mathcal{A}_{n}(x) \\
= & \frac{n_{f}}{16 \pi^{2}} c[D] \epsilon_{\mu \nu \lambda \sigma} F_{\mu \nu}^{a}(x) F_{\lambda \sigma}^{b}(x+\hat{\mu}+\hat{\nu}) \operatorname{tr}\left\{t^{a} t^{b}\right\}
\end{aligned}
$$

This is the particular solution of $\mathcal{A}_{n}(x)$. The complementary solution to the homogeneous equation $\sum_{x} \mathcal{A}_{n}(x)=0$ in general can be written as

$\mathcal{A}^{(h)}(x)=\sum_{\mu}\left[G_{\mu}(x)-G_{\mu}(x-\hat{\mu})\right] \equiv \partial_{\mu} G_{\mu}(x)$

where $G_{\mu}(x)$ is any local and gauge invariant function. Then the general solution of the anomaly function is the sum of the complementary solution and the particular solution,

$$
\begin{aligned}
& \mathcal{A}_{n}(x) \\
= & \frac{n_{f}}{16 \pi^{2}} c[D] \epsilon_{\mu \nu \lambda \sigma} F_{\mu \nu}^{a}(x) F_{\lambda \sigma}^{b}(x+\hat{\mu}+\hat{\nu}) \operatorname{tr}\left\{t^{a} t^{b}\right\} \\
& +\sum_{\mu}\left[G_{\mu}(x)-G_{\mu}(x-\hat{\mu})\right]
\end{aligned}
$$

In general, $G_{\mu}(x)$ depends on the background gauge field and $D$. Only when $\partial_{\mu} G_{\mu}(x)$ vanishes identically for any smooth gauge background, $D$ can reproduce the correct chiral anomaly provided $c[D]=1$. ( e.g., the Neuberger operator [8] ). On the other hand, the pathologies of the standard Wilson-Dirac operator are $c[D]=0$ and $\mathcal{A}^{(h)}(x) \neq 0$.

Now we consider $D$ with $\partial_{\mu} G_{\mu}(x)=0$ in a constant gauge background. Then we introduce local fluctuations to the background gauge field and/or increase its topological charge $Q$. Then $c[D]$ may remain constant provided that the local fluctuations of the gauge fields are not too violent and/or $Q$ is not too large. When the gauge background becomes so rough that $D$ is non-local, then $\partial_{\mu} G_{\mu}(x) \neq 0$ but the index theorem may still hold with the same $c[D]$. If we keep on increasing the roughness of the gauge background, then $D$ will undergo a topological phase transition, and $c[D]$ will become another integer or even a fraction [4].

\section{REFERENCES}

1. S. Adler, Phys. Rev. 177 (1969) 2426; J.S. Bell and R. Jackiw, Nuovo Cimento 60 (1969) 47.

2. G 't Hooft, Phys. Rev. Lett. 37 (1976) 8; Phys. Rev. D17 (1976) 3432; Phys. Rep. 142 (1986) 357.

3. T.W. Chiu and S.V. Zenkin, Phys. Rev. D59, 074501 (1999).

4. T.W. Chiu, hep-lat/9906007.

5. P. Ginsparg and K. Wilson, Phys. Rev. D 25 (1982) 2649.

6. T.W. Chiu, Phys. Lett. B445 (1999) 371.

7. T.W. Chiu, hep-lat/9810002, to appear in Phys. Rev. D.

8. H. Neuberger, Phys. Lett. B417 (1998) 141; Phys. Lett. B427 (1998) 353. 